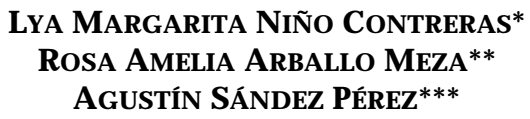

\title{
EVOLUCIÓN DEL SECTOR MANUFACTURERO BAJACALIFORNIANO DURANTE LA TRANSICIÓN HACIA LA APERTURA ECONÓMICA (1975-1993): UNA PERSPECTIVA SISTÉMICA
}

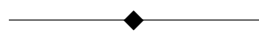

\section{RESUMEN}

Se examinan rasgos que caracterizan el comportamiento industrial del sistema deciudades bajacaliforniano duranteel periodo comprendido entre 1975 y 1993. Como ha sido usual, dada su importancia económica y social, el análisis se enfoca al sector de las manufacturas. Entre varios aspectos, destaca la nueva asignación de roles entre grupos industriales manufactureros (GIM), con una profundización en cambios que fueron gestándose desde fines de los setenta. En este sentido, se reconoce en la técnica de "cambio y participación" un método particularmente útil para señalar tales desplazamientos. El corte espacial utilizado, apoyado en una visión sistémica, se ha empleado antes como criterio de agregación (CONEPO, 1988), en tanto que sus objetivos resultan funcionales desde el punto de vista de la economía regional. A su vez, el corte temporal focaliza una coyuntura económica especialmente relevante, si consideramos los resultados observados en este mismo lapso en el empleo manufacturero a escala nacional.

Palabras clave: Baja California, Industrialización, Economía regional, Manufacturas, Cambio y Participación.

\footnotetext{
* Investigadora del instituto de investigaciones sociales de la universidad AUTÓNOMA DE BAJA CALIFORNIA. BECARIA PROMEP. Correo electrónico: Inino@uabc.mx ** INVESTIGADORA DEL INSTITUTO DE INVESTIGACIONES SOCIALES DE LA UNIVERSIDAD AUTÓNOMA DE BAJA CALIFORNIA. Correo electrónico: arballo@uabc.mx

* * INVESTIGADOR DEL INSTITUTO DE INVESTIGACIONES SOCIALES DE LA UNIVERSIDAD AUTÓNOMA DE BAJA CALIFORNIA. BECARIO PROMEP. Correo electrónico: sandez@uabc.mx

Artículo recibido en septiembre de 2003.

Artículo aprobado en octubre de 2003.
} 


\section{ABSTRACT}

This article studies some of the main economic figures of the industrialization process in system of cities of Baja California within the historic period from 1975 to 1993. Due to its economic relevance, the analysis is focused on the manufacturing sector. A new role is developing among industrial groups with growing changes that began in the late seventies. In this analysis the "shift \& share" technique is still useful to identify that kind of industrial behavior. This level of aggregation was used before (CONEPO, 1988) in order to achieve some objectives in accordance with a regional economics scope. At the same time, this period of analysis brings some light into a recent economic phase of special interest, taking into consideration main figures in the national manufacturing sector of the same period.

Keywords: Baja California, Industrialization, Regional Economics, Manufacturing Sector, Shift \& Share A nalysis.

\section{INTRODUCCIÓN ${ }^{1}$}

Entre los estudios regionales que se han propuesto esclarecer la naturaleza y evolución delaindustria en la frontera norte de México, hace más de una década que se cuenta con acercami entos que configuran el comportamiento económico del sector manufacturero en Baja California (Sández, 1987; Ortega, et al., 1995). En ellos se ofrece una visión longitudinal, comprendiendo varias fases en los rasgos característicos de un proceso de industrialización para el caso de esta región fronteriza. Es así como se elaboró una reseña de las trayectorias económicas para grupos industriales en localidades que conforman al sistema de ciudades. Destacan particularidades que este proceso asume en una experiencia marcada por el relativo aislamiento geográfico dela primera mitad del siglo pasado, seguido de una serie de acciones y programas promovidos por instancias gubernamentales, después de los años sesenta, con los que se buscó establecer condiciones para el crecimiento y lageneración de empleos.

\footnotetext{
${ }^{1}$ Se agradece a CONACYT por el apoyo proporcionado para la realización de los estudios de maestría como becaria Núm. 45484. Actualmente becaria Promep para realizar estudios de doctorado en Ciencias Sociales.
} 
La aplicación de la técnica de "cambio y participación" seconvierte en una herramienta útil para señal ar desplazamientos y establecer el tipo decomposición industrial (industrial mix), medida en empleo, a partir de que son disponibles estadísticas industriales regional es. Estecorte espacial, apoyado en un análisis desistema deciudades, seha empleado antes como criterio deagregación (CONEPO, 1988), en vista de su conveniencia y funcionalidad desde el punto de vista de la economía regional.

Las razones de efectuar un estudio para esteperiodo, comprendido entre 1975y 1993, son varias. Por una parte, secuenta con un antecedente en lo relativo al comportamiento integral del sistema deciudades bajacaliforniano (CONEPO, 1988), conformado por más de dos décadas de actividad económica. Además, se cuenta con algunas aplicaciones previas de la técnica de "cambio y participación" (shift \& share) orientadas al estudio del proceso de industrialización en esa fase(Sández, 1987; Ortega, et al ., 1995). El periodo analizado constituye para la economía mexicana una fase transicional entre el fin del desmantelamiento de algunos mecanismos proteccionistas y un esquema de liberalización para la apertura comercial.

En el caso de regiones como esta, vale mencionar que la operación del régimen de excepción que representa la zona libre, ha significado un factor que matiza considerablemente comportamientos, tanto industriales como comerciales. Por lo mismo, resulta relevante examinar particularidades en este proceso al presentarse en condiciones económica y geográficamente específicas, como sería el caso de una zona fronteriza.

La política industrial nacional se ha orientado principalmente al apoyo a la industria maquiladora. Tan sólo en el estado de Baja California, en 1993 se registraron 4099 establecimientos manufactureros con una participación al producto interno bruto regional del orden de 14.57\% (Secretaría de Desarrollo Económico de Baja Cal ifornia, 1996:4-31). Sin embargo, en crisis recientes el sector industrial ha sido afectado tanto en el ámbito nacional como en el regional. En 1986, la manufactura registró un decrecimiento de $-7.2 \%$ y de $-5.5 \%$, con leve recuperación a principios de los 
Estudios Fronterizos, Nueva época, vol. 4, núm. 7, enero-junio de 2003

noventa, para nuevamentedescender (Méndez, 1997:136). Por otro lado, en este largo periodo y principalmente en años recientes, se registraron acontecimientos que repercutieron en forma directa o indirecta en la industria manufacturera del sistema, entre los que deben considerarse:

- Dentro del proceso deglobalización económica, el ingreso de México al Acuerdo sobreA ranceles y Comercio (GATT) (1986); la implementación del tratado trilateral de libre comercio (1994); adel gazamiento del Estado antela implementación de políticas de ajuste estructural (1983-1989), ${ }^{2}$ crisis económicas de las décadas de los ochenta y noventa, y como respuesta a estas, cambios en la política monetaria, cambiaria e industrial. En el periodo posterior a la primera crisis se han identificado dos subperiodos: el de1982-1987, el cual secaracterizó por una liberación comercial gradual, y el de 1988-1994, definido por una liberación acelerada ${ }^{3}$ y una estrategia de industrialización orientada hacia exportaciones (Dussel, 1995); asimismo, se registraron cambios a la ley deinversión extranjera en 1989 y 1993.

- Los embargos estadounidenses en 1980 y 1991, han afectado en forma particular a la industria atunera, ${ }^{4}$ y de manera general al sector pesquero, situación que repercute directamente en Ensenada. Los embargos también han tenido graves consecuencias en el empleo: de acuerdo con reportes de la

2 La desincorporación comenzó en 1983 y se profundizó en 1989; el número de paraestatales se redujo de 1155 en 1982, a 210 a fines de 1993, y de 1989 a 1993 la privatización generó 23700 millones de dólares (Dussel, 1995).

${ }^{3}$ La liberación de importaciones pretendía entre otros objetivos fomentar la industrialización orientada a las exportaciones, de manera que el promedio arancelario disminuyó de $28.5 \%$ en 1985 a 12.5\% en 1992 (Dussel, 1995).

${ }^{4}$ El atún dejó de ser uno de los principales renglones del sector pesquero de expor-tación y una fuente importante de divisas. De 1991 a 1994 las ventas externas a nivel nacional sufrieron una severa caída al pasar de 58368 toneladas a 9302 lo que en términos de divisas significó una pérdida de un poco más de 250 millones de dólares (Cámara Nacional de la Industria Pesquera). 
Cámara Nacional de la Industria Pesquera, Ensenada perdió 7 mil empleos en el sector desde la declaración del embargo en 1991.

- En el plano de infraestructura en comunicaciones, la ampliación en la cobertura delíneas tel efónicas aumentó 164\% en el periodo de 1987 a 1993 (A nuario Estadístico de Baja Cal ifornia), así como la proliferación de los medios de comunicación por medio de microondas y satélites, fenómeno que pudo haber facilitado la promoción de las ventajas locacional es de la manufactura del sistema de ciudades.

Valeseñalar la inexistencia de un amplio consenso en cuanto al impacto de las crisis económicas en la frontera norte. De acuerdo con ciertos estudios, debido a su contenido económico internacional, la frontera norte registró una crisis más acentuada que el resto del país, lo que se reflejó en un mercado cambiario demasiado inestable. En este contexto, el impacto de la crisis se manifestó en forma dramática y con mayor intensidad en la región fronteriza (Ramírez, 1988:26). De acuerdo con cifras del Instituto Nacional de Estadística Geografía e Informática (INEGI) (1984), la tasa de inflación en la frontera norte durante 1981 fue de $25.3 \%$, de $66.3 \%$ para 1982, y de 168\% para 1983.

Otros autores han identificado al gunos efectos positivos, donde, a pesar dela situación de crisis eincertidumbre, la maquiladora se ve favorecida gracias a la disminución acelerada en costos de producción debido a la devaluación del peso respecto al dólar, sobre todo en lo concerniente a mano de obra y energéticos (Ramírez y Mungaray, 1985:11).

De cualquier modo, en este debate no se conocen aún estudios sectoriales específicos a partir de la fase de transición, concernientes a su posible repercusión en la evolución del empleo manufacturero, en el nivel degruposindustriales, para esteámbito y tampoco en el regional.

Es así que con intención de aportar elementos para el entendimiento de la evolución económica del sistema de ciudades, se estudian la dinámica y estructura manufacturera en el sistema respecto 
al comportamiento global y, a su vez, del sistema en su conjunto respecto a la evolución del empleo industrial nacional durante el periodo 1975-1993. Tales resultados ofrecen información útil en la reflexión acerca del proceso económico recientea la luz dela nue va visión depolítica industrial y comercial orientada a la apertura económica.

Cabeseñal ar quela perspectiva sistémica no representa en modo al guno una fetichización or reificación del espacio, en el sentido planteado antes por algunos autores (Coraggio, 1983), pues se asume quelas regiones por sí sol as no son agentes de cambio de los procesos industriales que tienen lugar en éstas. Por el contrario, tales procesos son sólo expresión territorial deactores sociales y desus relaciones. Asimismo, se reconoce la pertinencia de una identificación social de la estructura interna, dinámica y tendencias del empleo manufacturero, complementándose, en forma interdisciplinaria, con estudios cuya finalidad sea explicativo de factores que dieron lugar a tales cambios, como la globalización, los mercados de trabajo, el papel empresarial, entre otros.

El presente trabajo se sustenta en una teoría general de los sistemas, de modo que a continuación se retoman algunas aportaciones a los estudios de sistemas de ciudades; en un segundo momento, aparece un apartado concerniente a la técnica de análisis regional shift and share (versión síntesis) aplicada a la estructura del empleo dela industria manufacturera; en un tercero, al gunos antecedentes sobre la aplicación del enfoque de sistémico; en un cuarto momento, la caracterización del sistema de ciudades como objeto de estudio, para posteriormente anal izar estructura y dinámica industrial partiendo de lo general a lo particular por unidad de análisis y, por último, presentar conclusiones con base en re sultados.

Debido a la concurrencia de cambios importantes en la actividad económica y específicamente del sector manufacturero ${ }^{5}$ en

\footnotetext{
${ }^{5}$ La clasificación económica utilizada correspondea la empleada en estos periodos en el censo industrial, siendo ésta la que se utiliza a lo largo de los diversos cuadros estadísticos considerados en el presente análisis.
} 
Niño, Arballo y Sández/ Evolución del sector manufacturero

este largo periodo - 1975-1993 - y dada la disponibilidad de información, serealiza un cortetemporal y seanal izan dos subperiodos: 1975-1985 y 1985-1993. Es así queseutilizan porcentajes, tasas de crecimiento promedio anual y la técnica de análisis regional shift and share que proporciona elementos para conocer el comportamiento manufacturero regional con respecto al nacional, permitiendo comparar patrones de crecimiento deuna región respecto a cualquier unidad de análisis de mayor tamaño.

La distribución porcentual, así como el componente estructural derivado de la técnica de cambio y participación, permiten conocer cambios y tendencias en la estructura del empleo manufacturero, además de las tasas de crecimiento promedio anual y el

Clasificación económica en el sector manufacturero. SUBSECTOR 31 PRODUCTOS ALIMENTICIOS, BEBIDAS Y TABACO.

A LIM EN TOS. RAM A : 3111 Industria dela carne; 3112 Elaboración de productos lácteos; 3113 Elaboración de conservas alimenticias. Incluye concentrados para caldos (excluye las de carne y leche exclusivamente); 3114 Beneficio y molienda de cereales y otros productos agrícolas; 3115 Elaboración de productos de panadería; 3116 M olienda de nixtamal y fabricación de tortillas; 3117 Fabricación de aceites y grasas comestibles; 3118 Industria azucarera; 3119 Fabricación de cocoa, chocolate y artículos de confitería; 3121 Elaboración de otros productos alimenticios para el consumo humano; 3122 Elaboración de alimentos preparados para animales. BEBID AS. RA M A : 3130 Industria de las bebidas.

SUBSECTOR 32 TEXTILES, PRODUCCIÓN DE VESTIDO E INDUSTRIA DEL CUERO.

TEXTILES. RAM A :3212 Hilado; tejido y acabado de fibras blandas; excluye de punto; 3213 Confección con materiales textiles; incluye fabricación de tapices y alfombras de fibras blandas; 3214 Fabricación de tejidos de punta. VESTID O. RA M A : 3220 V estido. SUBSECTOR 38 PRODUCTOS METÁLICOS, MAQUINARIA Y EQUIPO, INCLUYE INSTRUMENTOS QUIRÚRGICOS Y DE PRECISIÓN.

PRODUCTOS METÁLICOS. RAMA: 3811 Fundición y moldeo de piezas metálicas ferrosas y no ferrosas; 3812 Fabricación de estructuras metálicas, tanques, calderas industriales, incluso trabajos de herrería; 3813 Fabricación y reparación de muebles metálicos; 3814 Fabricación de otros productos metálicos: MAQUINARIA Y EQUIPO ELÉCTRICO. RAMA: 3823Fabricación y/ o ensamble de maquinas de oficina, cálculo y procesamiento informático; 3831 Fabricación y/ o ensamble de maquinaria, equipo y accesorios eléctricos (incluye la generación de energía eléctrica); 3832 Trabajo y ensamble de equipo eléctrico de radio, televisión, comunicación y de uso médico; 3833 Fabricación y/ o ensamble de aparatos y accesorios de uso doméstico (excluye electrónicos); EQUIPO DE TRANSPORTE. RAMA: 3842 Fabricación y/ o ensamble de equipo detransporte y sus partes (excluye automóviles y camiones). 
Estudios Fronterizos, Nueva época, vol. 4, núm. 7, enero-junio de 2003

componentediferencial: el primero completa el conocimiento dela dinámica manufacturera por rama de actividad en sí misma, mientras que el segundo revela su comportamiento respecto al registrado en la industria nacional.

MARCO TEÓRICO-TÉCNICO REFERENCIAL. TEORÍA GENERAL DE SISTEMAS

La teoría general de sistemas es un cuerpo teórico del cual se desprende el enfoque de sistema de ciudades. Propone un lenguaje unificado que descubra isomorfismos o similitudes de estructura entre fenómenos y procesos de distintas ciencias (Racionero, 1981:16).

Bertalanffy (1956), un exponente clásico, señaló que existen modelos, principios y leyes que se aplican a sistemas generalizados o a subclases, independientemente de su clase particular, de la naturaleza de sus elementos componentes y de las relaciones o fuerzas entre ellos. Parece legítimo pedir una teoría, no de sistemas de una clase más o menos especial, sino de principios universales aplicables a los sistemas en general. De este modo llegamos a postular una nueva disciplina llamada teoría general de sistemas.

Bajo esta perspectiva se define como sistema al conjunto de objetos, más las relaciones entre estos y sus atributos (Hall y Fajen, 1956), aplicar esteconcepto al ámbito urbano-regional resulta que un sistema de ciudades o sistema urbano es el conjunto de ciudades más las relaciones que se ejercen entre estas y sus atributos.

Debido a su complejidad, el enfoque de ciudades se apoya en aportaciones dela economía regional y dela geografía urbana para el análisis de los sistemas urbanos. Para efectos de este estudio, las contribuciones corresponden a la teoría del lugar central de Christaller, a la regla rango-tamaño de Zipf, y a la teoría de difusión deinnovaciones deHermansen (CONAPO, 1991:84-86). Sin embargo, en vista dequela aplicación de esta teoría general desistemas a los estudios desistemas deciudades es relativamentereciente, se vuelvemucho más relevantela capacidad heurística o deinventiva 
del investigador respecto a la selección y definición delos atributos del objeto deestudio.

\section{ENFOQUE DE SISTEMAS DE CIUDADES}

Los estudios bajo el enfoque desistema de ciudades íntimamente serelacionan con la planificación urbano-regional, ya que proporcionan elementos que permiten aproximarnos a las manifestaciones del proceso deurbanización, esto es, la organización jerárquica y la distribución espacial de la estructura de los asentamientos humanos y económicos que lo conforman, así como desus interrelaciones con otros procesos (Graizbord y Garrocho, 1987; Garrocho, 1988). Lo anterior permiteinfluir o dirigir de algún modo la organización delos asentamientos humanos, mismos quecondicionan la forma en que se dan y se transmiten especialmente las relaciones económicas y la difusión o diseminación de innovaciones.

No obstante, a pesar desu importancia, el enfoque de sistemas de ciudades como instrumento de análisis de los sistemas urbanos se reveló hasta los años sesenta. Esto se debió principalmente a dos factores: por un lado, el rápido avance tecnológico de las computadoras en la década de los cincuenta, que permitió el de sarrollo de métodos de análisis cuantitativo como simuladores urbanos que facilitaron la real ización de estudios urbanos-regional de tipo experimental (Berry, 1964:117-125). Por otro lado, la publicación de las obras Regions, Resources and Economic Growth (Perloff et al., 1960) y M etrópolis and Region deOtis D. Duncan, que presentan una nueva visión del paisaje económico y social, fundamentada en la íntima relación entre economía y geografía, así como en la posibilidad de que las ciudades fueran descritas en términos desistemas urbanos (Bourney Simmos, 1978:9-10).

En 1964, Brian Berry conduyeen su artículo “Cities as Systems within Systems of Cities", quela teoría urbana y sus modelos podían ser vistos como un aspecto de la teoría general de sistemas. Esto quiere decir que las ciudades pueden ser consideradas como sistemas, es decir, entidades conformadas como el ementos interdependientes, susceptibles deestudiarse en diferentes aspectos y 
Estudios Fronterizos, Nueva época, vol. 4, núm. 7, enero-junio de 2003

niveles: a) en cuanto a su estructura, funcionamiento y dinamismo, b) pueden dividirse para su análisis en una variedad desistemas.

\section{LA EXPERIENCIA PREVIA, LA APLICACIÓN DEL ENFOQUE DE SISTEMAS DE CIUDADES}

A pesar de que la aplicación del enfoque de sistemas a estudios urbano-regionales es rel ativamente nueva esta se ha efectuado en un número de países con fines distintos. Por citar algunos estudios de esta naturaleza, tenemos los estudios de caso realizados por la onu en Brasil, México, Perú, Venezuela, Argentina, Colombia y Jamaica, los cuales tienen por objetivo mejorar la administración municipal deciudades medias. También seencuentran los casos del sistema de ciudades de la región pampeña AzulOlavarría y Tandil, en A rgentina (Sassone, 1992); el estudio de la distribución espacial del sistema cooperativo de ferias de consumo familiar y su papel en el abastecimiento alimentario de la región centro-occidental deVenezuela (Jaspeelves, 1991), y el estudio del sistema de ciudades y ordenación del territorio español (Racionero, 1981), entreotros.

Asimismo, con el objeto de identificar las zonas con ventajas para el logro delos objetivos delas políticas dedesconcentración de la vida nacional en México, surgen los estudios de: Salazar (1984), quetienen por objetivo conocer el comportamiento demográfico y económico de las ciudades alternativas para, en un momento dado, canalizar la desconcentración; Gustavo Garza (1980), quien desarrolla un análisis exhaustivo de los principales sistemas en México; y del Consejo Nacional de Población (CONAPO) (1991), queelaboró el diagnóstico del sistema nacional deciudades y la distribución espacial dela población en México.

\section{TÉCNICAS DE ANÁLISIS REGIONAL SHIFT AND SHARE O CAMBIO Y PARTICIPACIÓN}

La técnica de anál isis regional supone que el comportamiento de la región en estudio debería mostrar el mismo ritmo decrecimiento 
que el de su referente, a menos que presente ventajas o desventajas comparativas. Esta se ha utilizado desde hace casi treinta años, y desde entonces ha recibido gran cantidad de aplicaciones (Brown, 1971; Parakeevopoulos, 1971; Floys y Sirmans, 1973; Stevens y Moore, 1980; Jamer Jr. y Hugehs, 1975; Bishop y Simpson, 1972). Asimismo, con el tiempo se han desarrollado varios enfoques de la técnica, a saber: la versión estadounidense; la inglesa; la de cuatro componentes y la de Bishop y Simpson (1972), siendo las primeras las más antiguas, así como las más utilizadas. Sin embargo, estas versiones presentan limitaciones en la interpretación de los componentes de cada rama de actividad, ya que a nivel agregado, los resultados son iguales, situación que se salva en la versión deBishop y Simpson (1972), la cual es una síntesis entre las versiones estadounidensee inglesa, donde se da un tratamiento más fino a la información. En ella, se estandarizan aspectos estructurales y dinámicos de la actividad industrial, reduciendo deficiencias de otras versiones.

Es así que para efectos de este estudio se aplica el enfoque propuesto por Bishop y Simpson (1972). Esta técnica proporciona elementos para conocer el comportamiento de la economía regional bajo la composición dela economía nacional, ya que mediante ésta es posible comparar los patrones de crecimiento de una región con cualquier unidad que sea mayor. Para este estudio se aplica al sistema/ nación, región valle/ sistema y región costa/ sistema en los periodos 1975-1985 y 1985-1993. Además, la técnica descompone al cambio total en tres elementos: estos son el componente nacional, el componente estructural y el componente diferencial. Para efectos deeste estudio se analizan los componentes estructural y diferencial.

Componente estructural: Refleja la composición industrial; permiteidentificar el rol deindustrias básicas; cuando el resultado es positivo y grande para algún sector, indica que la región ha encontrado ventajas locacional es en especial ización en esa actividad (Sández, 1987).

Componente diferencial o dinámico: Se refiere a la posición competitiva de la región; el aumento en el cambio diferencial ocurre 
porque al gunas industrias crecen en al gunas regiones más rápido que en otras; las tasas nacionales de crecimiento de estas industrias son más altas que la tasa de crecimiento nacional. Regiones caracterizadas por industrias crecientes son áreas en las cuales las ventajas geográficas para actividades particulares han mejorado en relación con otras regiones. El incremento en este componente se debe a que industrias regionales crecen mas rápido que esas mismas industrias a nivel nacional, es decir, permiteidentificar el grado de motricidad que al canzan estas (Sández, 1987).

Es importante subrayar que la técnica de cambio y participación por no tratarse de una teoría del crecimiento, no pretende algún poder explicativo, mas ofrece una interpretación clara de cambios en la estructura y dinámica industrial y del empleo en el crecimiento regional (Stilwell, 1970), resultados que se complementan con otros análisis macroeconómicos y cualitativos.

\section{Sistema de ciudades Tijuana-Mexicali-San Luis Río Colorado. Antecedentes}

En 1989, en México, con el fin de identificar zonas con ventajas o potencial para el logro de objetivos de las políticas de desconcentración de la vida nacional, el conA Po elaboró un diagnóstico del sistema nacional deciudades y distribución espacial de la población mexicana. De dicho estudio se deriva que el sistema nacional deciudades, de acuerdo con la figura 1, seconforma por 79 lugares central es, así como por 31 sistemas de ciudades (CONAPO, 1991), (González y Pang, 1993:47-49). El sistema de ciudades Tijuana-M exicali-San Luis Río Colorado es uno de los 31 sistemas de ciudades que se detectaron en dicho estudio.

A partir del flujo telefónico y carretero registrado se puede hablar de un sistema de ciudades integrado en su configuración por núcleos complementarios de organización sectorial y especialmente diferentes en cuanto a su clima, dinámica demográfica, económica, en cuanto a recursos disponibles y potencial mente aprovechables (CONEPO, 1998). 
Figura 1. Sistema Nacional de Ciudades de México, estructura jerárquica y vínculos.

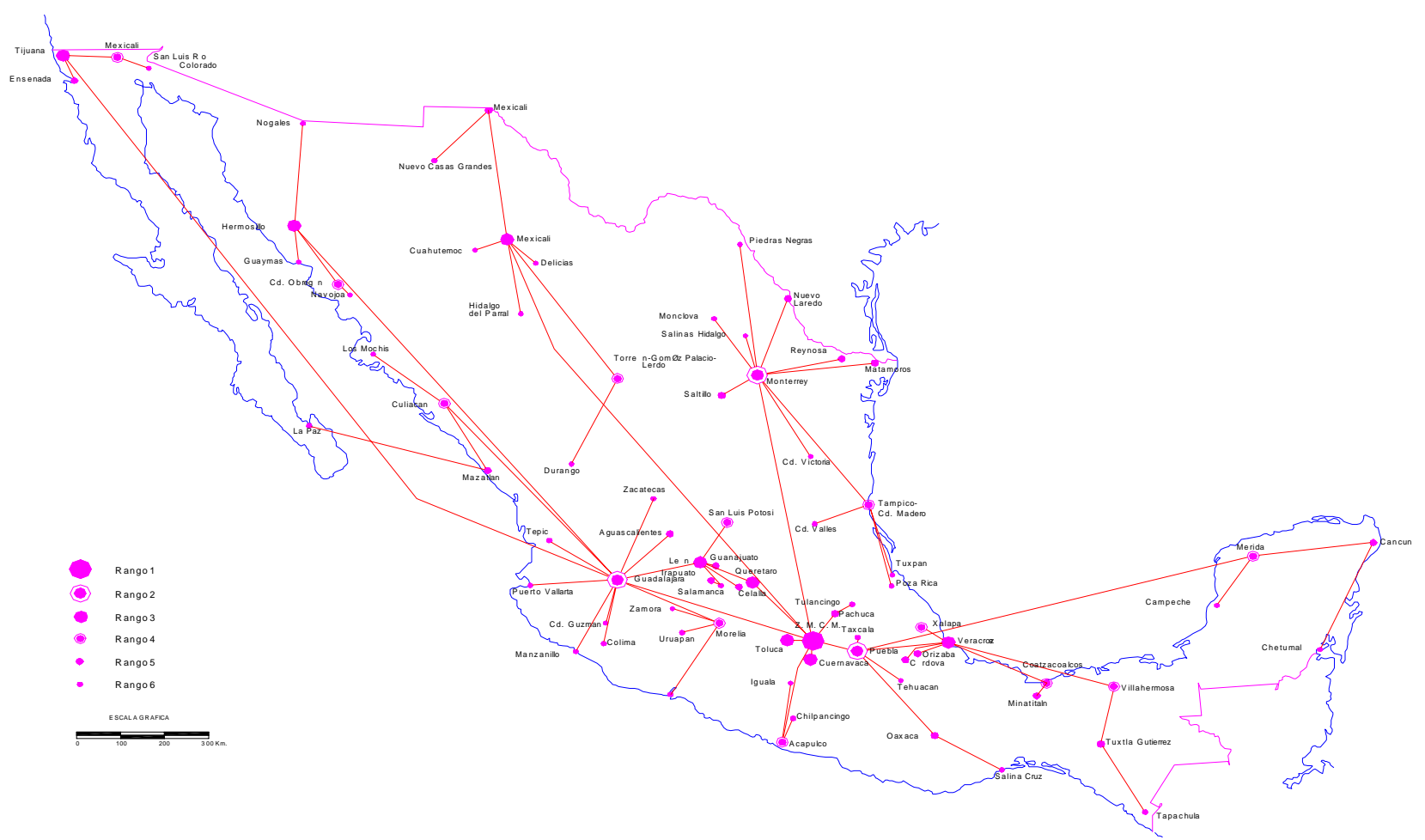

Fuente: Conapo (1991) Sistema de ciudades y distribución espacial de la población en México, Vol. 1. 
El sistema se conforma por seis cabeceras municipales: ${ }^{6}$ Ensenada, Tijuana, Playas de Rosarito, Tecate, Mexicali y San Luis Río Colorado, las cuales se localizan especial mente en la franja fronteriza México-Estados Unidos -excepto Ensenada-, situación que a través del tiempo las ha llevado a presentar una dinámica distinta al referente nacional (CONEPO, 1988) (ver figura 2). Asimismo estas poblaciones se caracterizan por su dimensión internacional, por su gran dinamismo geográfico, económico y social, por su al to grado de urbanización, así como por su desvinculación económica, política y social con respecto al resto de la república mexicana7 (Alegría, 1992:63).

El sistema se localiza en el extremo noroeste del país y comprende el estado de Baja Cal ifornia y parte del estado de Sonora. Al norte colinda con los Estados Unidos y al sur con el estado de Baja California Sur, sus límites corresponden a los paralelos 32 grados 43 minutos y 28 grados delatitud norte; al estecolinda con el estado de A rizona (EUA), con el municipio de Puerto Peñasco, Sonora, y con el Golfo deCalifornia, y al oestecon el océano Pacífico, entre los meridianos 112 grados 48 minutos y 117 grados 8 minutos de longitud oeste (SPP en CONEPO, 1988:3).

El sistema está integrado por los municipios deBaja California: Ensenada, Mexicali, Tecatey Tijuana más el municipio sonorense deSan Luis Río Colorado. Esteúltimo municipio fueincorporado al sistema de ciudades en estudio a partir de un criterio funcional con dos vertientes: la primera radica en el al to grado de integración funcional que presenta el municipio de San Luis Río Colorado con el de Mexicali, al compartir la explotación de los recursos

\footnotetext{
${ }^{6}$ Actualmente el sistema se conforma por seis cabeceras municipales, ya que la delegación de Playas de Rosarito que hasta 1995 había formado parte del municipio de Tijuana, se elevó a rango de cabecera municipal a principios de 1996. Sin embargo, aún no se cuenta con información para el municipio de Rosarito, por lo que para efectos de este estudio no contempla a Rosarito como municipio, sino como una localidad perteneciente al municipio de Tijuana.

${ }^{7}$ Prueba de ello son un sinfín de programas federales que se han realizado con el objetivo de vincular la frontera norte con el interior del país, los cuales datan desde 1851 con el Programa de Zonas y Perímetros Libres (Mendoza B., 1982:46-47).
} 
Figura 2. Sistema de Ciudades Tijuana-M exicali-San Luis Río Colorado.

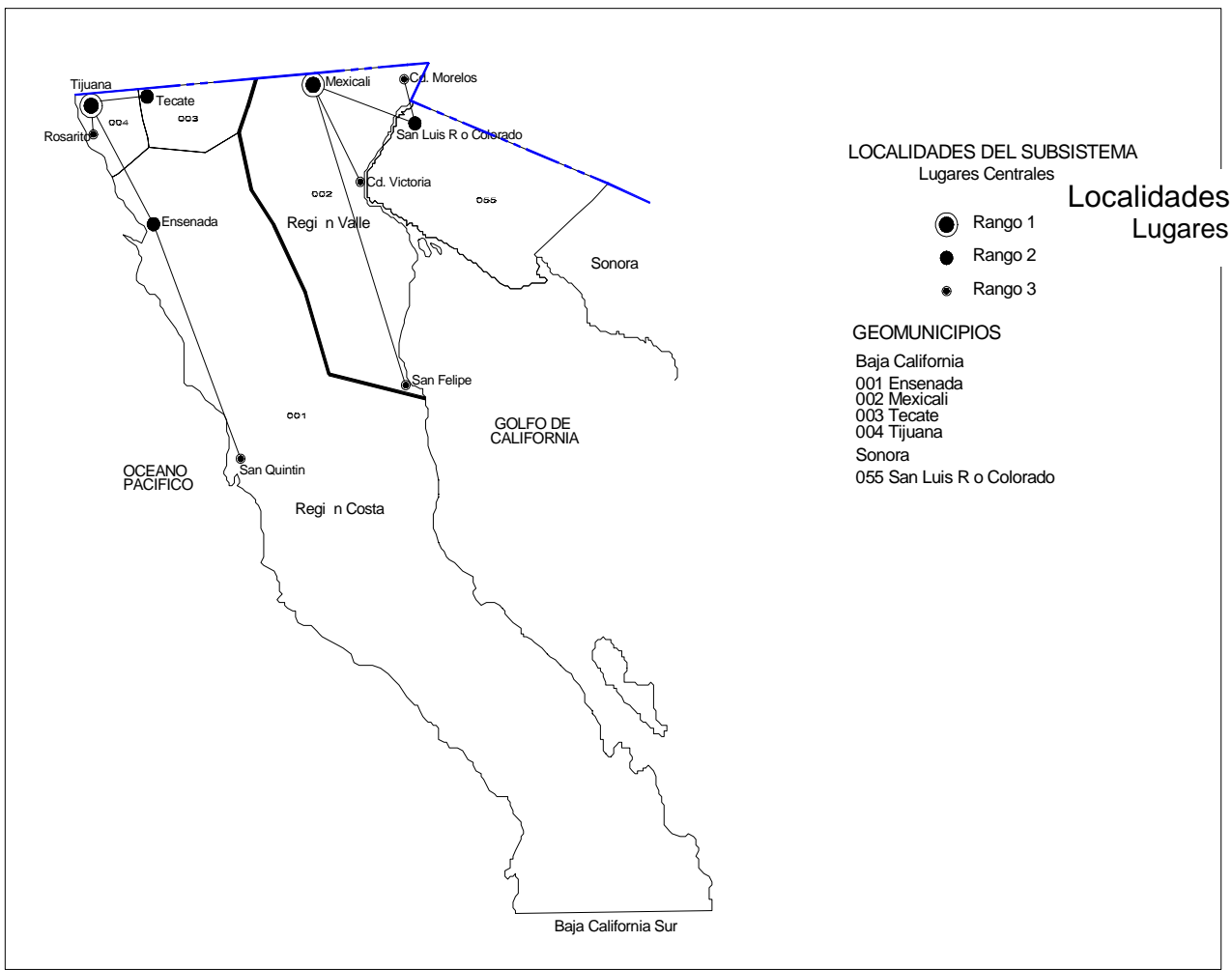

FUENTE: Sistema de ciudades y distribución espacial de la población en México, Vol.

1, CONAPO (1991). 
naturales del distrito deriego del Río Colorado, actividad significativa en lo que respecta a la ocupación, ingreso y producto del municipio sonorense. La segunda, sesustenta fundamentalmente en el relativo aislamiento funcional y geográfico de San Luis Río Colorado respecto al resto del estado de Sonora, al mediar entre ellos el desierto de Altar (CONEPO, 1988:3-5).

De acuerdo con el sentido, al cance y límites de la interacción del sistema a partir del análisis de flujos carreteros y telefónicos, el estudio del CONEPO establece que el sistema está integrado por dos regiones funcional mente diferentes: denominadas "Ia región costa", que se articula por la ciudad de Tijuana y tiene bajo su área de influencia a Ensenada, Tecate, Rosarito, San Quintín; y la "región valle", quese articula por Mexicali y tiene bajo su área de influencia a Estación Victoria, Ciudad M orelosy demás pequeñas localidades del valle, así como a San Felipey San Luis Río Colorado (CONAPO, 1991:402) (ver figura 3).

\section{INDUSTRIA MANUFACTURERA DEL SISTEMA DE CIUDADES POLÍTICAS DE DESARROLLO REGIONAL}

Desdemediados desiglo seregistraron varios acontecimientosque fueron alterando la dinámica económica, demográfica y social en el sistema de ciudades. Entre estos están la caída del precio internacional del algodón, la salinización de las tierras del valle de Mexicali, el cese dela segunda guerra mundial y dela ley seca, así como del programa de braceros, querepatrió aproximadamentea 348764 connacional es, donde una partede estos terminó por asentarse en ciudades que conforman el sistema.

En este marco de deterioro económico, el sistema, hasta entonces, había basado su economía en el sector primario y terciario; prueba deello esqueen 1930, el 81.13\% dela población económicamente activa deM exicali y Tijuana se concentraba en el sector primario, y el restante, $18.87 \%$, sedistribuía en los sectores secundario y terciario. Tal situación hacía convenientediversificar su economía, lo que sehabía hecho patentecon la debacle del sector primario y con este del terciario, ocasionando un al to grado de desempleo. 
FIGURA 3. Jerarquía y principales vínculos entre las localidades del sistema deciudades TijuanaMexicali-San Luis Río Colorado.

\begin{tabular}{|l||l||l||l}
\hline Rango 1 & Rango 2 & Rango 3 & $\begin{array}{l}\text { Relaci n con } \\
\text { localidades de }\end{array}$ \\
\hline
\end{tabular}
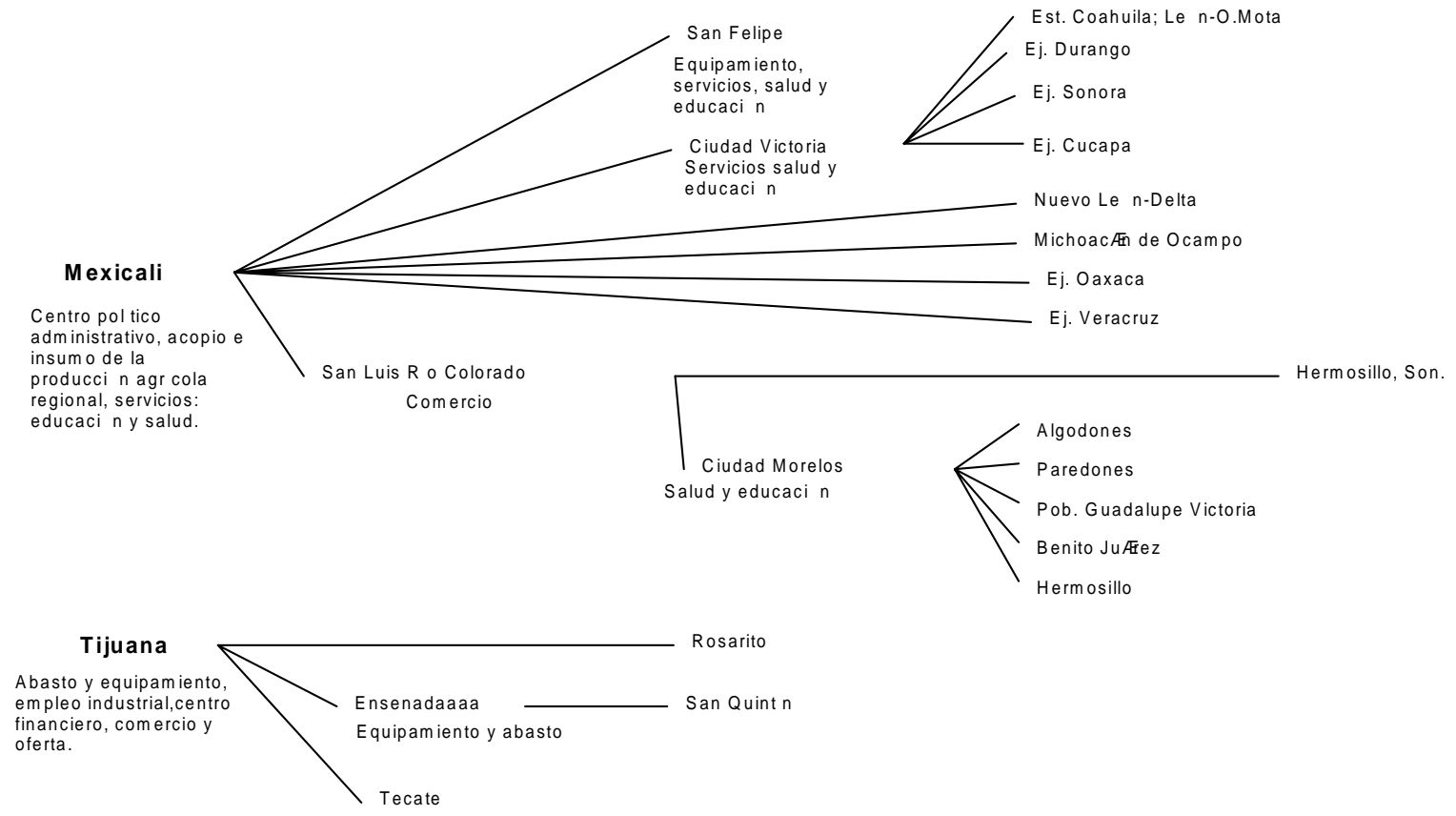

Fuente: conapo (1991). Sistema de ciudades y distribución espacial de la población en México, Vol. 1, conapo (1991). 
Es así como las dificultades por incorporar población desempleada a la economía regional, y aprovechando bondades del régimen dezona libre, el cual tiene como antecedenteal régimen de perímetros libres - primera política de desarrollo regional implementada en la frontera norte — el gobierno mexicano implementó el Programa Nacional Fronterizo 1961-1965(Mendoza B., 1982:52) $\mathrm{y}$, posteriormente, el programa de la industria maquiladora, de 1965-1975, en dos fases.

El primer programa tenía como objetivo general, fomentar económica y socialmente el desarrollo de la región y “mostrar al extranjero un M éxico apegado a la realidad y a la excelencia de sus auténticos valores" (Bermúdez, Programa Nacional Fronterizo, México, 1961 en: Mendoza B., 1982:52). El segundo programa, en su primera fase, tenía por objetivo la creación deempleos, elevación deingreso y el nivel devida dela población fronteriza, formación demano deobra clasificada, eincorporación deinsumos nacionales a la producción delas plantas maquiladoras (Mendoza B., 1982:52).

En la segunda fase del programa, a partir de 1975, anteel rezago persistente en la reducción del número de empresas establecidas, escasa producción de artículos para satisfacer las demandas del mercado regional, entreotros, secreó el programa para fomentar el desarrollo industrial de la zona fronteriza, cuyos objetivos consistían en: eliminar las causas del rezago; impulsar la industrialización de productos agropecuarios; satisfacer las necesidades de la población; estimular las exportaciones de artículos elaborados en la zona; y sustituir las importaciones de artículos terminados por materias primas y generar empleos para la población (Mendoza B., 1982:64-65).

De esta manera el modelo de desarrollo económico primarioterciarizado - base económica del sistema de ciudades- ya configurado en el periodo 1900-1960, seconsolidó con el impulso dado al sector secundario, básicamenteen áreas urbanas que seconvertirían en polos de atracción migratoria, especialmente M exicali y Tijuana, con el Programa Industrial Fronterizo (Castillo, 1980-84), donde todos los programas industriales tenían como constante la integración dela frontera nortedel mercado nacional. 
Posterior a la crisis económica de 1982 se dio un parteaguas entre los objetivos de la política económica industrial fronteriza. Es así quea partir del programa de desarrollo dela frontera norte de 1985, los objetivos se encontraban cada vez más sujetos a vicisitudes de la nueva política económica, de la apertura del mercado mundial, ${ }^{8}$ de libre comercio y de las transformaciones del Estado benefactor en normativo, pero también a los cambios sustanciales del tipo decambio, reestructuración industrial y comercio externo (García, 1990:61). Lo anterior se evidencia con el desplazamiento de la integración nacional a segundo término, mismo quehabía sido objetivo principal y recurrente en los programas anteriores.

Los cambios registrados en los programas industriales fronterizos a partir de mediados de los ochenta tenían por objeto sentar las bases para el proceso de homologación arancelaria. Es así que en 1993 se firmaron convenios que permitirían la transición del régimen especial de zona libre y franja fronteriza al régimen de desgravación arancelaria implícita en el Tratado de Libre comercio de A mérica del Norte (TLCAN) (Alarcón, 1995:65). Con estegiro en política económica industrial seharían extensivas las "ventajas del libre comercio" al resto del país, dinámica que desde principios de siglo ya se experimentaba en la frontera norte.

\section{DINÁMICA DE LA INDUSTRIA MANUFACTURERA} (1975-1993)

Debe considerarse que, siendo el periodo en estudio bastante extenso, decasi veinteaños, en él, la evolución dela economía mexicana se caracteriza por su notable transformación. Por ello, en el periodo 1970-1990 se identifican cuatro fases del cicl o económico en la economía mexicana ${ }^{9}$ (García, 1992:30).

\footnotetext{
${ }^{8}$ Se crearon programas para el fomento de las exportaciones como Programa de Importación Temporal (PITEX) y el Programa deEmpresas AltamenteExportadoras (ALTEX), (Dussel, 1995:464).
} 
Estudios Fronterizos, Nueva época, vol. 4, núm. 7, enero-junio de 2003

Las fases económicas deBaja California parecen no diferenciarsemucho del ciclo nacional, aunque existan algunas a destacar. En la primera fase, el crecimiento es más sostenido; durante la fase de auge la tasa de crecimiento medio anual es más sostenida y de mayor altura; la crisis impactó menos a la entidad, con excepción de 1985; y por último aparece que la entidad sufre una recesión mayor quela nación (García: 1992-38).

\section{ANÁLISIS DE CAMBIO Y PARTICIPACIÓN ${ }^{10}$}

Se identifican dos tendencias en la manufactura del sistema de ciudades. Por un lado, desde 1975 viene incrementánd ose la participación porcentual de cinco de once grupos industriales aquí señalados. Los grupos que incrementan su importancia relativa son: maquinaria y equipo eléctrico; maquinaria no eléctrica; hule y plástico; muebles no metálicos, y productos no metálicos, pasando de $29.3 \%$ a $55.6 \%$.

En la región costa destaca, por un lado, la expansión del grupo maquinaria y equipo —el cual llega a generar poco más deun tercio

${ }^{9}$ La fase de crecimiento, 1970-1976, se caracterizó por una tasa de crecimiento media anual (тсмA) positiva; la de auge, 1977-1981, un crecimiento sostenido y de mayor altura de la fase anterior; la de crisis, 1982-1986 con, тсм A negativa; y la de estancamiento o recesión, de 1987-1990. La fase de estancamiento es cuando la TсMA es constante, y la de recesión cuando disminuye de un año a otro pero sin llegar a ser negativa (García Montaño, 1992:30-37).

${ }^{10}$ Notación utilizada para la aplicación de la técnica:

Cambio total:

Componente nacional:

Componente estructural:

Componente diferencial:

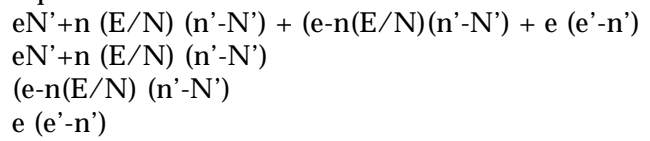
e $=$ Empleo de la industria i en la región en el año base.
$\mathrm{E}=$ Empleo total industrial en la región en el año base.
$\mathrm{n}=\quad$ Empleo de la industria $\mathrm{i}$ en la nación en el año base.
$\mathrm{N}=\quad$ Empleo total industrial en la nación en el año base.
$\mathrm{e}^{\prime}=\quad$ Tasa de crecimiento del empleo en la industria i regional.
$\mathrm{E}^{\prime}=\quad$ Tasa de crecimiento del empleo total industrial regional.
$\mathrm{n}^{\prime}=\quad$ Tasa de crecimiento del empleo de la industria i nacional.
$\mathrm{N}^{\prime}=\quad$ Tasa de crecimiento del empleo total industrial nacional. 
CUADRO1. Composición de la industria manufacturera en el sistema TMSL*(1975-1993).

\begin{tabular}{|c|c|c|c|c|c|c|c|c|c|}
\hline \multirow{2}{*}{$\begin{array}{l}\text { Grupo Industrial } \\
\text { Manufacturero (GIM) }\end{array}$} & \multicolumn{3}{|c|}{ Empleo sistema } & \multicolumn{3}{|c|}{ Región valle } & \multicolumn{3}{|c|}{ Región costa } \\
\hline & 1975 & 1985 & 1993 & 1975 & 1985 & 1993 & 1975 & 1985 & 1993 \\
\hline Alimentos & 28.5 & 17.9 & 11.5 & 20.4 & 17.1 & 17.5 & 33.2 & 18.4 & 9.3 \\
\hline Bebidas & 3.6 & 9.2 & 2.6 & 2.2 & 2.8 & 2.6 & 4.4 & 5.0 & 2.6 \\
\hline Vestido & 9.6 & 5.1 & 3.8 & 12.0 & 8.7 & 6.4 & 8.1 & 2.7 & 2.8 \\
\hline Muebles no metálicos & 2.8 & 4.5 & 6.2 & 3.0 & 4.4 & 4.1 & 2.7 & 4.6 & 7.0 \\
\hline Química & 1.6 & 1.3 & 0.8 & 2.5 & 2.3 & 1.5 & 1.1 & 0.6 & 0.5 \\
\hline Hule y plástico & 0.6 & 3.0 & 8.5 & 0.2 & 1.1 & 3.7 & 0.8 & 4.2 & 10.3 \\
\hline Minerales no metálicos & 4.1 & 3.8 & 3.8 & 1.8 & 5.0 & 4.7 & 5.6 & 3.1 & 3.5 \\
\hline Productos metálicos & 6.8 & 8.5 & 7.7 & 5.4 & 10.8 & 10.7 & 7.6 & 7.1 & 6.6 \\
\hline Maquinaria no eléctrica & 1.8 & 5.3 & 2.1 & 2.8 & 2.2 & 1.7 & 1.1 & 7.3 & 2.3 \\
\hline Maq. y equipo eléctrico & 23.3 & 22.8 & 31.3 & 21.1 & 21.4 & 25.9 & 24.6 & 23.8 & 33.3 \\
\hline Equipo de transporte & 5.4 & 4.2 & 2.8 & 14.5 & 7.6 & 8.8 & 0 & 2 & 0.5 \\
\hline Subtotal & 88.1 & 85.6 & 81.2 & 86.1 & 83.3 & 87.6 & 89.2 & 78.7 & 78.9 \\
\hline Otros & 11.9 & 14.4 & 18.8 & 13.9 & 16.7 & 12.4 & 10.8 & 21.3 & 21.1 \\
\hline TOTAL & 100 & 100 & 100 & 100 & 100 & 100 & 100 & 100 & 100 \\
\hline
\end{tabular}

FUENTE: Elaboración propia a partir de información de Censos Económicos, INEGI, varios años.

* Tijuana, Mexicali, San Luis Río Colorado. 
CUADRo 2. Técnica de cambio y participación. Componente estructural de la manufactura en sistema TMSL*, 1975-1993.

\begin{tabular}{|c|c|c|c|c|c|c|}
\hline \multirow{2}{*}{$\begin{array}{c}\text { Grupo Industrial } \\
\text { Manufacturero (GIM) }\end{array}$} & \multicolumn{2}{|c|}{ Empleo sistema } & \multicolumn{2}{|c|}{ Empleo región valle } & \multicolumn{2}{|c|}{ Empleo región costa } \\
\hline & (1975-1985) & (1985-1993) & (1975-1985) & (1985-1993) & (1975-1985) & (1985-1993) \\
\hline Alimentos & -760.4 & 158.6 & 660.1 & 168.8 & -660.1 & -168.8 \\
\hline Bebidas & 15.9 & 5.9 & -46.7 & 304.6 & 46.7 & -304.6 \\
\hline Vestido & -499.7 & 384.9 & -254.6 & -541.7 & 254.6 & 541.7 \\
\hline Muebles no metálicos & 106.3 & 340.7 & 25.9 & 20.5 & -25.9 & 20.5 \\
\hline Química & -824.8 & 2294.8 & -43.6 & -219.3 & 43.6 & 219.3 \\
\hline Hule y plástico & -284.4 & -166.3 & -316 & -2078 & 316 & 2078 \\
\hline Minerales no metálicos & 125.2 & -76.4 & 40.9 & -5.1 & -40.9 & 5.1 \\
\hline Productos metálicos & 43.3 & 169.5 & -75.8 & -123.6 & 75.8 & 123.6 \\
\hline Maquinaria no eléctrica & 191.2 & -237.8 & 470.7 & 1083.5 & -470.7 & -1083.5 \\
\hline Maquinaria y equipo eléctrico & 3918 & 2848.1 & 9.7 & -312.9 & -9.7 & 312.9 \\
\hline Equipo de transporte & 27.4 & 219.1 & -447.7 & -673 & 447.7 & 673 \\
\hline
\end{tabular}

FUENTE: Elaboración propia a partir de información de Censos económicos, INEGI, varios años.

* Tijuana, Mexicali, San Luis Río Colorado. 
del empleo-; así como el incremento - aunqueen menor proporción y más reciente — delos grupos huley plástico y muebles nometálicos. Cabeseñalar queestos tres grupos concentran $50.6 \%$ del empleo; además, cada uno de ellos registra participación porcentual mayor respecto a la manufactura total del sistema, lo quesignifica un grado de especialización en estos grupos.

Por otro lado, se registra una caída constante en la participación del resto delos gruposindustriales, dondeel más afectado es el dealimentos, el cual desciendesu participación de 33.2\% a 9.3\% en el periodo de 1975 a 1993.

En la región valle, en cambio, sólo cuatro grupos incrementaron su importancia relativa, principal mente productos metálicos y minerales no metálicos, los cuales, al estar por arriba de la participación general del sistema, muestran condiciones de especialización: los otros son maquinaria y equipo eléctrico, en menor medida, muebles y ligeramente, el grupo bebidas. Cabedestacar queel descenso específico en la participación relativa del grupo alimentos no es tan marcada en el valle como en la región costa, al pasar de $20.4 \%$ a $17.5 \%$.

Los grupos industriales que ofrecen mayores ventajas de especialización en el sistema son química, maquinaria y equipo eléctrico, y en menor medida, vestido y muebles no metálicos. Entre los menos favorecidos seencuentran maquinaria no eléctrica, hule y plástico, y minerales no metálicos. En la región costa la mayor parte de los grupos presentan ventajas; los más altos son vestido, maquinaria y equipo eléctrico, hule y plástico, y transporte. De acuerdo con estos resultados, la región cuenta con ventajas en su especialización.

En general, entre los grupos desfavorecidos se encuentran maquinaria no eléctrica, mi neral es no metálicos y al imentos. Cabe señalar que, aunque el último presente ventajas locacionales negativas, estas se atenúan respecto al periodo anterior; ello pudiera relacionarse con la importancia que han adquirido tanto el Valle de la Trinidad como San Quintín en Ensenada. En cambio, en la región valle, si bien presentan ventajas positivas, estas disminuyen respecto al periodo anterior. 
CUADRO 3. Técnica de cambio y participación. Componente diferencial de la manufactura en el sistema TMSL, 1975-1993.

\begin{tabular}{|lcrrrrr|}
\hline $\begin{array}{c}\text { Grupo Industrial } \\
\text { Manufacturero (GIM) }\end{array}$ & \multicolumn{2}{c}{$\begin{array}{c}\text { Empleo sistema } \\
\text { (1975-1985) }\end{array}$} & $\begin{array}{c}\text { Empleo región valle } \\
\text { (1985-1993) }\end{array}$ & $\begin{array}{c}\text { Empleo región costa } \\
\text { (1975-1985) }\end{array}$ & $\begin{array}{c}\text { (1985-1993) } \\
\text { (1975-1985) }\end{array}$ & (1985-1993) \\
\hline Alimentos & -2.2 & 1499.4 & 1139.6 & 622.9 & -1139.6 & -622.9 \\
Bebidas & -209.2 & 778.4 & 85.0 & 30.1 & -85.0 & -30.1 \\
Vestido & -698.0 & 1581.0 & 631.3 & -1218.8 & -631.3 & 1218.8 \\
Muebles no metálicos & 205.5 & 248.4 & -36.2 & -1897.4 & 36.2 & 1897.4 \\
Química & -71.3 & 235.8 & 96.4 & -233.8 & -96.4 & 233.8 \\
Huley plástico & -1309.8 & -675.5 & -25.4 & -265.7 & 25.4 & 265.7 \\
Minerales no metálicos & 830.2 & 1470.7 & 796.0 & -1032.5 & -796.0 & 1032.5 \\
Productos metálicos & 8797.4 & 2520.1 & 1001.8 & -1392.6 & -1001.8 & 1392.6 \\
Maquinaria no eléctrica & -905.9 & -40.5 & -1362 & 161.3 & 1362.0 & -161.3 \\
Maq. y equipo eléctrico & - & 7800.5 & 422.3 & -6679.3 & -422.3 & 6679.3 \\
Equipo de transporte & - & -524.5 & -712.0 & 595.0 & - & -595.0 \\
TOTAL & 882.1 & 15809.3 & 72.0 & -13800.5 & -72 & 13800.5 \\
\hline
\end{tabular}

FUENTE: elaboración propia a partir de información de Censos económicos, INEGI, varios años. 
Al parecer, la región valle resulta más vulnerable que la región costa, ya que para la mayor parte de los grupos manufactureros descienden sus ventajas de especialización; sólo maquinaria y equipo no el éctrico y bebi das incrementaron sus ventajas de un periodo a otro.

El componente diferencial permite medir el grado de motricidad de la industria manufacturera. Tanto la manufactura del sistema de ciudades en su conjunto como la de la costa incrementan su posición competitiva de un periodo a otro; es decir, el empleo manufacturero del sistema creció más rápido que el nacional y el de la región costa fue más dinámico que el del sistema y, por lo mismo, que el de la nación.

Cabe resaltar el desempeño de la industria manufacturera de la región costa, ya que en el primer subperiodo de análisis había crecido por debajo del empleo manufacturero del sistema. En la región valle sucede lo contrario: su posición competitiva pasa de positiva a negativa en el segundo periodo; es decir, creció menos que el empleo manufacturero en su conjunto.

En la región valle sólo los grupos equipo de transporte y maquinaria no eléctrica incrementan su posición competitiva de un periodo a otro; en cambio, en la región costa, son estos mismos, junto con alimentos y bebidas los que no mejoran tal característica.

Los resultados confirman los obtenidos antes, en los que, duranteel primer subperiodo, el empleo manufacturero dela región vallemuestra mayor dinamismo que la región costa, mientras que en el empleo del sistema, ya en un segundo subperiodo, seobserva un cambio en esta correlación, en la que la generación del empleos de la costa fue mucho más dinámica, registrando ritmos de crecimiento por arriba de la manufactura total del sistema. En este sentido, la región valle presenta crecimientos muy por debajo de la dinámica del resto.

En la manufactura del sistema en su conjunto, vestido y maquinaria no eléctrica son los únicos grupos que presentan descenso respecto al primer periodo. En la región costa son alimentos, bebidas, maquinaria no eléctrica y equipo detransporte, mientras que 
CUADRO 4. Tasa de crecimiento manufacturero en el sistema TMSL* (1975-1993).

\begin{tabular}{|lccccrr|}
\hline $\begin{array}{c}\text { Grupo Industrial } \\
\text { Manufacturero (GIM) }\end{array}$ & $\begin{array}{c}\text { Sistema empleo } \\
\text { (1975-1985) }\end{array}$ & $\begin{array}{c}\text { Empleo región valle } \\
\text { (1985-1993) }\end{array}$ & \multicolumn{2}{c|}{$\begin{array}{c}\text { Empleo región costa } \\
\text { (1975-1985) }\end{array}$} \\
\hline (1985-1993) & (1985) & (1985-1993) \\
\hline Alimentos & 1.6 & 6.3 & 5.2 & 7.5 & 0 & 5.5 \\
Bebidas & 8.0 & 5.8 & 9.6 & 6.1 & 7.5 & 5.6 \\
Vestido & -0.1 & 8.2 & 3.7 & 3.2 & -5 & 15.4 \\
Muebles no metálicos & 11.6 & 16.8 & 11.2 & 6.2 & 11.8 & 21.1 \\
Química & 3.9 & 6.1 & 6 & 1.9 & 0.2 & 13.1 \\
Huley plástico & 26.0 & 27.8 & 24.8 & 25.3 & 26.2 & 28.2 \\
Minerales no metálicos & 5.6 & 12.1 & 18.5 & 6.2 & 0.1 & 16.6 \\
Productos metálicos & 8.9 & 10.9 & 14.7 & 7.1 & 5.4 & 13.9 \\
Maquinaria no eléctrica & 18.9 & 0.2 & 4.3 & 3.7 & 27.9 & -0.6 \\
Maq. y equipo eléctrico & 6.3 & 16.8 & 7.2 & 9.8 & 5.8 & 19.8 \\
Equipo de transporte & 3.8 & 6.7 & 0.4 & 9.1 & 92.9 & -2.2 \\
\hline TOTAL & 6.5 & 12.2 & 7.1 & 7.2 & & 6.1 \\
\hline
\end{tabular}

FUENTE: Elaboración propia con información de Censos económicos, INEGI, varios años.

* Tijuana, Mexicali, San Luis Río Colorado. 
en la región valle, esto sucede sólo en los grupos dealimentos, hule y plástico, maquinaria y equipo el éctrico, y equipo detransporte.

\section{CONSIDERACIONES FINALES}

La dinámica regional registrada en el pasado reciente junto con cambios en políticas económicas eindustriales impactaron en forma heterogénea localidades y regiones que conforman el sistema deciudades bajacaliforniano. En particular, seobserva un desplazamiento de al gunos grupos industriales, sobre todo los más tradicionales, en términos de su comportamiento en el empleo manufacturero. Lo anterior resulta comprensible dada su evolución a largo plazo y grado relativo de especialización de subre giones que componen al sistema. Esta diferencia, sin embargo, se acentúa acorde con una correlación económica, desplazando la región costa a la región valle, la cual, aún en un primer subperiodo (1975-1985), presentaba mayor dinamismo en generación de empleos quela primera.

La política industrial orientada hacia la manufactura se aprecia en forma clara durante el segundo subperiodo, principalmente en la región costa, en donde transforma significativamente al paisaje industrial. Del mismo modo, se aprecia concentración de mano deobra en grupos industriales demaquinaria y equipo eléctrico, y en menor medida más recientemente en los grupos hule y plástico, y muebles no metálicos, concentrando $50.6 \%$ del empleo en la región costa y $33.7 \%$ en la región valle.

Vale destacar el dinamismo productivo de la región costa si se considera que, a pesar de la etapa recesiva del ciclo económico en quela entidad se ve más afectada que la nación, esta región el evó su ritmo degeneración deempleo. Ello seasocia seguramentea la fuerte presencia de los grupos industriales de maquinaria y equipo eléctrico y, en menor medida, los grupo huley plástico, y muebles no metálicos.

Resultados como estos, a medida que ofrecen evidencia de mejores oportunidades y ventajas de especialización en el caso de industrias no tradicionales eintensivas en tecnología flexibley uso 
demano deobra debaja y mediana calificación, hacen recomendable examinar un conjunto derivado de implicaciones sociales, laborales, incluyendo términos de legislación ambiental, daños a la salud, factores de riesgo, infraestructura y servicios, niveles de bienestar, organización laboral, organización empresarial, vocaciones industriales y económicas, para cada subregión y localidad en el sistema.

\section{BiBLIOGRAFíA}

Alarcón, Diana. 1995. “Librecomercio y homologación arancelaria en las zonas libres y franjas fronterizas de México" en: Frontera norte, vol. 7, núm. 14, julio-diciembre, El Colegio de la Frontera Norte, Tijuana, Baja California.

Alegría, Tito. 1992. D esarrollo urbano en la frontera M éxico-Estados U nidos, Consejo Nacional para la Cultura y las Artes, Colección Regiones, México, D.F.

Bertalanffy, Ludwig Von. 1956. G eneral Systems Theory, Year Book of the Society for the Advancement of General Systems Theory, vol. I.

Bishop, K. C. y C. E. Simpson. 1972. “Components of Change A nalysis: Problems of Alternative A pproaches to Industrial Structure" en: Regional Studies, vol. 6, Pergamon Press, Gran Bretaña.

Bourne, L. y J. W. Simmons. 1978. Systems of Cities. Readings on Structure, Growth, and Policy.

Castillo, Víctor. 1990. Economía fronteriza y desarrollo regional, UABC, Mexicali, Baja California.

Castillo, Víctor. 1991. “La configuración espacial del desarrollo regional fronterizo", en: Estudios Fronterizos, número 21, enero-abril, Instituto de Investigaciones Sociales-UABC, Mexicali, Baja California.

CONAPO. 1991. Sistema de ciudades y distribución espacial de la población en M éxico, vols. I y II, México. 
CONAPO-CONEPO. 1984. Estudio sociodemográfico del estado de Baja California, Baja California.

CONEPO. 1988. Estudio de sistema ciudades Tijuana-M exicali-San Luis Río Colorado", Mexicali, Baja California.

Coraggio, José Luis. 1983. "Social Spaceness and the Concept of Region, en Frank Moulaert, "Regional A nalysis and the International Division of Labor" , Klwer, Nijhoff Publishing, EUA.

Dussel, Enrique. 1995. “El cambio estructural del sector manufacturero mexicano, 1988-1994”, en: Comercio Exterior , vol. 45, núm. 6, México, junio.

Faerstein, Paul, et al. 1993. "Secondary Cities and Urban Management in Brazil. Historical and Institutional Framework", en:The M anagement of Secondary Cities in Latin A merica, United N ations Centre For H uman Settlements, pp. 113-132. 1993. "Case of Study. Angra Dos Reis, Brazil", en: The $M$ anagement of Secondary Cities in Latin A merica, United Nations CentreFor Human Settlements, pp. 118-133.

García M ontaño, Jorge. 1990. “Sector externo, crecimiento económico y bienestar en Baja California: 1970-1988”, en: Estudios Fronterizos, núm. 22, mayo-agosto, IIS-UABC, Mexicali, Baja California.

Garza, Gustavo. 1980. “La industrialización de las principales ciudades de M éxico. Hacia una estrategia espacio-sectorial de descentralización industrial” "El Colegio de México, México.

Garrocho, Carlos. 1988. "A nálisis del sistema de ciudades de San

Luis Potosí", tesis de maestría, El Colegio de México.

González, Ligia y Leticia Pang Molina 1993. “Sistema deciudades en México", en Ciudades, núm. 19, julio-septiembre, RNUI, México.

Graizbord, Boris y Carlos Garrocho. 1987. "Sistemas de ciudades:

Fundamentos teóricosy operativos", pp. 98(mimeo).

Hall, A. D. y Fagen, R. E. 1956. D efinition of Systems. Y ear Book of The

Society for The A dvancement of General System Theory, vol. 1.

INEGI. (1984). La inflación en la frontera.

INEGI. (1989, 1994). Censos Económicos, Baja California. 
Jaspe, Ismael y Paul Ives. 1991. “Estudio de la distribución espacial del sistema cooperativo de ferias de consumo familiar (FCF) y de su papel en el abastecimiento alimentario en la región centro-occidentedeVenezuela", en: R evista G eográfica, núm. 114, julio-diciembre, Instituto Panamericano de Geografía eHistoria.

Mendoza Barrueto, E. 1982. “Historia de los programas federales para el desarrollo económico dela frontera norte" en: Mario Ojeda (ed.), A dministración del D esarrollo dela Frontera $\mathrm{N}$ orte, Colegio de México, A.C., México, D.F.

Méndez, Silvestre. 1997. Problemas económicos de M éxico, 3a. ed., McGraw-Hill.

Ortega, Guadal upe, et al. (1992). “Componentes de cambio y economía regional: El sector manufacturero en el estado de Baja California y en el municipio de Mexicali (1985-1988)", en: Estudios Fronterizos, núms. 27-28, enero-abril/ mayo-agosto, IIS-UABC, Mexicali, Baja California.

Perloff, H.S. et al. 1960. Regions, Resources, and Economic Growth, Johns Hopkins, University Press.

Racionero, Luis. 1981. Sistema deciudades y ordenación del territorio, Alianza Universidad.

Ramírez Acosta, Ramón de Jesús. (1988). “Comportamiento del espacio financiero de la frontera norte de México en el contexto de la crisis económica. El caso de Tijuana, B.C.", en: E conomía, serie 3 , núm. 3, UABC.

Ramírez, Ramón dejesúsy Alejandro Mungaray. 1985. “El impacto dela crisis cambiaria de 1982 en las relaciones económicas fronterizas: El caso deTijuana-San Diego", en Economía, serie 1, núm. 2, UABC.

Salazar Ruíz, Hector. 1984. La dinámica de crecimiento de ciudades intermedias de M éxico. Los casos de León, San Luis P otosí y Torreón (1970-1980), El Colegio de México- Consejo Nacional de Población, pp. 106.

Sández Pérez, Agustín. 1987. “Estructura y dinámica del sector manufacturero bajacaliforniano (1960-1985)", en Cuadernos 
de ciencias sociales, serie 3, núm. 7, IIS-UABC, Mexicali, Baja California.

1987. Estadísticas básicas del sector manufacturero en Baja California (1960-1975), Serie Estadísticas Básicas, IIS-UABC, Mexicali, Baja California.

Sassone, Susana. 1992. "Sistemas urbanos policéntricos en los sistemas nacionales de ciudades. Un caso en la Argentina", en: R evista G eográfica, núm. 16, julio-diciembre, Instituto Panamericano de Geografía e Historia.

Somocurcio et al. 1993. "Case Study. Chiclayo, Peru", en: The $M$ anagement of Secondary Cities in Latin A meric, United N ations Centre For H uman Settlements, pp. 90-112.

Ruíz, Raúl. 1996. “Sección Nacional”, Semanario Z eta, Tijuana, Baja California.

Stilwell, F.J.B. 1970. "Further thoughts on the Shift and Share Approach", Regional Studies, vol. 4, Pergamon Press LTD, Gran Bretaña. 